\title{
Synthesis and Anti-Cancer Activities of Resveratrol Derivatives
}

\author{
Xing Zheng1,2*, Liuying Yu ${ }^{1,2 *}$, Xu Yao1,2, Bo Lv³, Zehua Yang1,2, Qutong Zheng1,2, \\ Haiying Duan 1,2,4, Chen Song1,2,4, Hailong Xie ${ }^{3 \#}$ \\ ${ }^{1}$ Institute of Pharmacy \& Pharmacology, University of South China, Hengyang, China \\ ${ }^{2}$ Hunan Province Cooperative Innovation Center for Molecular Target New Drug Study, Hengyang, China \\ ${ }^{3}$ Cancer Research Institute, University of South China, Hengyang, China \\ ${ }^{4}$ Research Interest Group of Pharmacy, University of South China, Hengyang, China \\ Email:"xhl0078@sina.com.cn
}

Received 13 August 2016; accepted 27 September 2016; published 30 September 2016

Copyright (C) 2016 by authors and Scientific Research Publishing Inc.

This work is licensed under the Creative Commons Attribution International License (CC BY).

http://creativecommons.org/licenses/by/4.0/

(c) (i) Open Access

\section{Abstract}

A novel series of resveratrol derivatives were synthesized according to Wittig-Horner reaction with 3,5-dihydroxybenzyl alcohol or 3,5-dimethoxybenzyl alcohol or 4-hydroxybenzyl alcohol as raw material and the inhibitory activities on breast carcinoma (MDA-MB-231) and gastric carcinoma cell lines (SGC-7901) in vitro were evaluated by the standard methyl thiazole tetrazolium (MTT) method. The result of biological test shows that some of resveratrol derivatives possess stronger anti-cancer activities than 5-FU. Compound $5 \mathrm{c}$ shows the strongest activity against breast carcinoma (MDA-MB-231) and gastric carcinoma cell lines (SGC-7901) with IC $_{50}$ value of $50.19 \pm$ $1.02 \mu \mathrm{M}, 122.68 .27 \pm 2.04 \mu \mathrm{M}$, compared to that $\mathrm{IC}_{50}$ value of $5-\mathrm{FU}$ is $98.59 \pm 3.61 \mu \mathrm{M}, 156.74 \pm 6.16$ $\mu \mathrm{M}$, respectively.

\section{Keywords}

Resveratrol Derivatives, Wittig-Horner Reaction, MTT Method, Breast Carcinoma, Gastric Carcinoma

\section{Introduction}

Cancer is the term used for diseases in which abnormal cells divide without control and are able to invade other tissues. All cancers begin in cells, when the DNA of a cell becomes damaged or changed, it will produce muta-

\footnotetext{
*These two authors contributed equally to this article.
}

${ }^{\#}$ Corresponding author.

How to cite this paper: Zheng, X., Yu, L.Y., Yao, X., Lv, B., Yang, Z.H., Zheng, Q.T., Duan, H.Y., Song, C. and Xie, H.L. (2016) Synthesis and Anti-Cancer Activities of Resveratrol Derivatives. Open Journal of Medicinal Chemistry, 6, 51-57. http://dx.doi.org/10.4236/ojmc.2016.63005 
tions that will affect normal cell growth and division. In recent years, cancer has become one of the main causes of death to the human being [1]. The incidences of breast cancer and gastric cancer are increasing rapidly. Breast cancer, the top cancer in women in the global, is particularly spreading in China at an amazing rate, and it has become a leading cause of cancer-related death worldwide [2]. In 2014, an estimated 235,030 new cases have been diagnosed, and 40,430 deaths from breast cancer occurred [3]. Gastric cancer is also one of the major causes of cancer death worldwide, with almost 990,000 cases detected annually. The incidences of gastric cancer vary with geographic location, and they are the highest in Eastern Asia including China, Japan and Korea [4]. Despite its prevalence, there is still no curative modality for late-diagnosed gastric cancer. The mechanism of carcinogenesis is complex and poorly understood. Nobili S. et al. [5] believed that gastric cancer appeared by the accumulation of both genetic and epigenetic changes, while de Souza C. R. et al. [6] said that it was influenced by both infection with Helicobacter pylori and genetic factors. The incident of cancer is higher and higher, and the treatment of cancer is imminent, so finding the best method for the treatment of cancer is the primary task for us. Chemotherapy is one of the major approaches of all the measures of cancer treatment. It has always been the focus in the research of anti-cancer candidates with high efficacy, low toxicity, and minimum side effects from nature plants.

Resveratrol (3,5,4'-trihydroxy-trans-stilbene, Figure 1) was originally isolated by Takaoka from the roots of hellebore in 1940 [7], and later, from the roots of Japanese knotweed in 1963, a plant used in traditional Chinese medicine. It attracted wider attention only in 1992 when its presence in wine was suggested as the explanation for cardioprotective effects [8]. Gradually, resveratrol was also found in Liliaceous, Polygonaceae, Leguminosae, Asmyrtaceae 21 families, 31 genera and 72 species of plants [9]. It was also reported to possess remarkable activities of anti-cancer [10], anti-bacterial [11], anti-aging [12], anti-inflammatory [13], and anti-oxidant [14]. The anti-oxidative and anti-inflammatory effects of resveratrol play a critical role in the therapeutic processing, evidence has revealed that resveratrol acts as a free radical scavenger; it promotes nitric oxide production, increases HDL cholesterol, and inhibits platelet coagulation and vasodilation [15]. These biological effects may explain the strange phenomenon "French paradox", a decreased incidence of cardiovascular diseases in moderate consumers of red wines despite an intake of a high-fat diet [16].

Although resveratrol possesses a series of pharmacological activities, its therapeutic application is still limited due to its short biological half-life (8 - 14 min) [17]. Experiments have proved that the enterohepatic recirculation and rapid first pass metabolism lead to its poor systemic bioavailability [18]. Since the last few years, significant progress has been made in studying the biological effects of resveratrol and the analogues. Cushman $\mathrm{M}$. et al. [19] tested the activity of 70 different resveratrol analogues as aromatase for chemotherapy cancer. The aromatase inhibitory activities of some analogues were much more effective than the lead compound resveratrol. From the structure-activity relationship of resveratrol study, it was proved that the lipophilic groups introduced in the structure help to improve the bioactivity. With this concept in mind, we introduce lipophilic group to ring A or B, and alkylate the instability of the hydroxyl in order to provide lead compounds for independent innovation of anti-cancer drugs by designing and synthesizing a series of new and trifluoromethyl resveratrol derivatives and evaluating the anti-cancer to breast carcinoma (MDA-MB-231) and gastric carcinoma cell lines (SGC7901).

\section{Materials and Methods}

${ }^{1} \mathrm{H}$ NMR spectra were recorded with an Agilent Technologies 400/54 Premium shielded spectrometer (400 MHz). ${ }^{19} \mathrm{~F}$ NMR spectra were recorded with an Agilent Technologies $400 / 54$ Premium shielded $(376 \mathrm{MHz}) .{ }^{13} \mathrm{C}$ NMR spectra were recorded with an Agilent Technologies 400/54 Premium shielded (101 MHz) spectrometer.

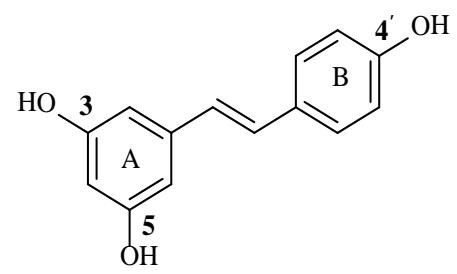

Figure 1. Structure of resveratrol. 
MS was recorded with a Hewlett-Packard HP-5989A spectrometer. Infrared spectra were measured with a Perkin-Elmer 983 spectrometer. Melting point was detected by DSC-Q2000. Unless otherwise noted, reagents were commercially available analytical grade materials used as supplied, without further purification.

General procedures for the preparation of compounds $\mathbf{5 a} \sim 5 \mathbf{c}$

The synthetic route of resveratrol derivatives $\mathbf{5 a} \sim \mathbf{5 c}$ is shown in Scheme 1. To obtain the product via fourstep reaction by using commercially available 3,5-dimethoxylbenzyl alcohol as the starting material. First, the raw material $(2.5 \mathrm{~g}, 15 \mathrm{mmol})$ was dissolved in DCM $(15 \mathrm{~mL})$ and stirred at $0^{\circ} \mathrm{C}$, then a solution of phosphorous bromide $(1.5 \mathrm{~mL}, 16 \mathrm{mmol})$ in the presence of DCM $(10 \mathrm{~mL})$ was added drop wise at the condition of ice-salt bath for $2 \mathrm{~h}$. The resulting mixture was poured into ice-water $(40 \mathrm{~mL})$, separating the organic layer, washing it with saturated brine to neutral $\mathrm{pH}$. Dried and evaporated solvent under vacuum. The mixture was filtered through silica gel to get a white needle crystal compound 2 (3,5-dimethoxybenzyl bromide), yield $85 \%$. A solution of compound $2(1.5 \mathrm{~g}, 6.5 \mathrm{mmol})$ and triethylphosphite $(1.5 \mathrm{~mL}, 8.7 \mathrm{mmol})$ was stirred at $130^{\circ} \mathrm{C}$ for $5 \mathrm{~h}$. Vacuum distilling to remove excess triethylphosphite to get colorless oil compound $\mathbf{3}$ (3,5-dime-thoxybenzyl phosphonate). Next, in the same reacting three-necked bottle, after sodium methanolate $(1.1 \mathrm{~g}, 20.5 \mathrm{mmol})$ in DMF $(7 \mathrm{~mL})$ stirring at $0^{\circ} \mathrm{C}$ for $30 \mathrm{~min}$, the fluorine-substituted benzaldehyde $\mathbf{4 a} \sim 4 \mathbf{c}(6.6 \mathrm{mmol})$ was added under ice-salt bath condition for $2 \mathrm{~h}$ and room temperature over night. The resulting mixture was poured into ice-water $(30 \mathrm{~mL})$, white solid precipitation appeared and then it was washed till neutral and recrystallize with ethyl alcohol (95\%) to get white crystal compound $\mathbf{5 a} \sim 5 \mathbf{c}$.

General method for synthesis of compounds 10a - 10c

Compounds 10a $\sim 10 \mathrm{c}$ were obtained as shown in Scheme 2. A solution of hydroxy-substituted benzyl alcohol $(50 \mathrm{mmol})$ into acetone $(50 \mathrm{~mL})$ and potassium carbonate $(150 \mathrm{mmol})$ as base was stirred at room temperature for 30 min and then bromoalkane was added at refluxing temperature for $18 \sim 48 \mathrm{~h}$. The mixture was filtered to remove potassium carbonate. Filtrate was evaporated under vacuum to give the compound 7 (alkoxysubstituted benzyl alcohol). Longer alkyl chains resulted in longer reaction times. The next steps for these compounds 10a $\sim 10 \mathrm{c}$ were similar to the synthesis of compounds $\mathbf{5 a} \sim 5 \mathbf{c}$.

All the new compounds were characterized by detailed spectroscopic analysis.

5a (trans-3,5-dimethoxy-2'-fluoro-4'-methoxy stilbenes)

White solid, yield $31 \%$, m.p. $59.7^{\circ} \mathrm{C} \sim 60.3^{\circ} \mathrm{C}$. IR $v_{\max }\left(\mathrm{cm}^{-1}\right)$, 832, 962, 1032, 1066, 1290, 1457, 1506, 1597, 1620, 2837, 2938. ${ }^{19} \mathrm{~F}$ NMR (376 MHz, $\left.\mathrm{CDCl}_{3}\right) \delta-115.51$ (dd, $\left.J=12.5,8.8 \mathrm{~Hz}\right) .{ }^{1} \mathrm{H}$ NMR $\left(400 \mathrm{MHz}, \mathrm{CDCl}_{3}\right) \delta$ 7.50 (t, $J=8.8 \mathrm{~Hz}, 1 \mathrm{H}), 7.17$ (d, $J=16.5 \mathrm{~Hz}, 1 \mathrm{H}), 6.97$ (d, $J=16.5 \mathrm{~Hz}, 1 \mathrm{H}), 6.71$ (dd, $J=8.7,2.5 \mathrm{~Hz}, 1 \mathrm{H}), 6.66$ (d, $J=2.2 \mathrm{~Hz}, 2 \mathrm{H}), 6.63(\mathrm{dd}, J=12.6,2.5 \mathrm{~Hz}, 1 \mathrm{H}), 6.39(\mathrm{t}, J=2.2 \mathrm{~Hz}, 1 \mathrm{H}), 3.83(\mathrm{~s}, 6 \mathrm{H}), 3.82(\mathrm{~s}, 3 \mathrm{H}) .{ }^{13} \mathrm{C}$ NMR $\left(101 \mathrm{MHz}, \mathrm{CDCl}_{3}\right) \delta 162.32(\mathrm{~s}), 160.94$ (s), 160.30 (d, $\left.J=11.2 \mathrm{~Hz}\right), 159.84$ (s), 139.58 (s), 128.53 (d, $J=4.8$ $\mathrm{Hz}), 127.65$ (d, $J=5.6 \mathrm{~Hz}), 121.25$ (d, $J=3.1 \mathrm{~Hz}), 117.56$ (d, $J=12.5 \mathrm{~Hz}), 110.48$ (d, $J=2.9 \mathrm{~Hz}), 104.40$ (s), 101.58 (d, $J=26.0 \mathrm{~Hz}$ ), 99.91 (s), 55.47 (d, $J=22.7 \mathrm{~Hz}$ ). HRMS(EI), Calcd. for, $\mathrm{C}_{17} \mathrm{H}_{17} \mathrm{O}_{3} \mathrm{~F}$, 288.1162, Found, 288.1158 .<smiles>CCOP(=O)(Cc1cc(OC)cc(OC)c1)OCC</smiles><smiles>[R]c1ccc(/C=C/c2cc(OC)cc(OC)c2)cc1</smiles>

Scheme 1. Synthetic steps of compounds 5a 5c. Reagents and conditions, a) DCM, ice salt bath, 2 h to room temperature, $2 \mathrm{~h}$, b) $\left.130^{\circ} \mathrm{C}, 5 \mathrm{~h}, \mathrm{c}\right) \mathrm{DMF}$, MeONa, $0^{\circ} \mathrm{C}$ to room temperature, overnight. 


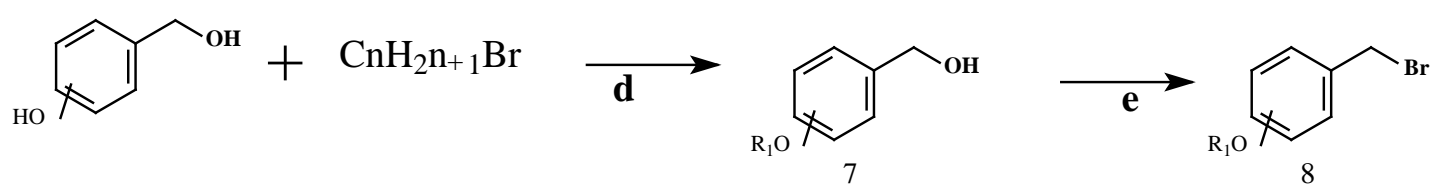

6<smiles>CCOP(=O)(Cc1ccc(C)cc1)OCC</smiles>

9<smiles>[R9]OC(C)(C)Cc1ccccc1CP(=O)(OCC)OCC</smiles>

9<smiles>[R2]c1cccc(C=O)c1</smiles>

Scheme 2. Synthetic steps of compounds 10a 10c. Reagents and conditions, d) Acetone, reflux, $18 \sim 48 \mathrm{~h}$, e) $\mathrm{PBr}_{3}, \mathrm{DCM}$, ice salt bath, $2 \mathrm{~h}$ to room temperature, $\left.\left.2 \mathrm{~h}, \mathrm{f}\right) 130^{\circ} \mathrm{C}, 5 \mathrm{~h}, \mathrm{~g}\right) \mathrm{DMF}, \mathrm{MeONa}, 0^{\circ} \mathrm{C}$ to room temperature, overnight.

\section{5b (trans-3,5-diethoxy-3'-fluoro-4'-methoxy stilbenes)}

White solid, yield $35 \%$, m.p. $59.9^{\circ} \mathrm{C} \sim 62.3^{\circ} \mathrm{C}$. IR $v_{\max }\left(\mathrm{cm}^{-1}\right)$, 683, 827, 958, 1065, 1155, 1204, 1459, 1518, 1591, 2838, 2937. ${ }^{19} \mathrm{~F}$ NMR (376 MHz, $\left.\mathrm{CDCl}_{3}\right) \delta-135.24$ (dd, $\left.J=12.4,8.5 \mathrm{~Hz}\right) .{ }^{1} \mathrm{H}$ NMR $\left(400 \mathrm{MHz}, \mathrm{CDCl}_{3}\right) \delta$ 7.29 (dd, 12.4, $8.5 \mathrm{~Hz}, 1 \mathrm{H}), 7.18(\mathrm{~d}, J=8.5 \mathrm{~Hz}, 1 \mathrm{H}), 6.98$ (d, $J=16.2 \mathrm{~Hz}, 1 \mathrm{H}), 6.92(\mathrm{~d}, J=8.5 \mathrm{~Hz}, 1 \mathrm{H}), 6.88(\mathrm{~d}$, $J=16.2 \mathrm{~Hz}, 1 \mathrm{H}), 6.64(\mathrm{~d}, J=2.2 \mathrm{~Hz}, 2 \mathrm{H}), 6.39(\mathrm{t}, J=2.2 \mathrm{~Hz}, 1 \mathrm{H}), 3.91(\mathrm{~s}, 3 \mathrm{H}), 3.83(\mathrm{~s}, 6 \mathrm{H}) .{ }^{13} \mathrm{C}$ NMR $(101$ $\left.\mathrm{MHz}, \mathrm{CDCl}_{3}\right) \delta 160.96(\mathrm{~s}), 153.74(\mathrm{~s}), 151.29$ (s), 147.29 (d, $\left.J=11.1 \mathrm{~Hz}\right), 139.15$ (s), 130.68 (d, $J=6.6 \mathrm{~Hz}$ ), 127.86 (s), 127.72 (d, $J=2.5 \mathrm{~Hz}), 123.02$ (d, $J=3.2 \mathrm{~Hz}$ ), 113.48 (s), 113.28 (d, $J=2.6 \mathrm{~Hz}), 104.43$ (s), 99.90 (s), 56.25 (s), 55.34 (s). HRMS(EI), Calcd. for, $\mathrm{C}_{17} \mathrm{H}_{17} \mathrm{O}_{3} \mathrm{~F}, 288.1162$, Found, 288.1158.

\section{5c (trans-3,5-diethoxy-4'-((trifluoromethyl)thio) stilbenes)}

White solid, yield 37\%, m.p. $96.1^{\circ} \mathrm{C} \sim 96.7^{\circ} \mathrm{C} .{ }^{19} \mathrm{~F}$ NMR (376 MHz, $\left.\mathrm{CDCl}_{3}\right) \delta-42.84$ (s, 3F). ${ }^{1} \mathrm{H}$ NMR (400 MHz, $\left.\mathrm{CDCl}_{3}\right) \delta 7.63(\mathrm{~d}, J=8.3 \mathrm{~Hz}, 2 \mathrm{H}), 7.54(\mathrm{~d}, J=8.3 \mathrm{~Hz}, 2 \mathrm{H}), 7.12(\mathrm{~d}, J=16.3 \mathrm{~Hz}, 1 \mathrm{H}), 7.06(\mathrm{~d}, J=16.3$ $\mathrm{Hz}, 1 \mathrm{H}), 6.68$ (d, $J=2.2 \mathrm{~Hz}, 2 \mathrm{H}), 6.43(\mathrm{t}, J=2.2 \mathrm{~Hz}, 1 \mathrm{H}), 3.84(\mathrm{~s}, 6 \mathrm{H}) .{ }^{13} \mathrm{C}$ NMR $\left(101 \mathrm{MHz}, \mathrm{CDCl}_{3}\right) \delta 161.01$ (s), 139.77 (s), 138.62 (s), 136.63 (s), 131.07 (s), 128.01 (s), 127.59 (s), 127.36 (s), 122.93 (d, $J=2.1 \mathrm{~Hz}$ ), 104.82 (s), 100.47 (s), 55.38 (s). HRMS(EI), Calcd. for, $\mathrm{C}_{17} \mathrm{H}_{15} \mathrm{~F}_{3} \mathrm{O}_{2} \mathrm{~S}$, 340.0745, Found, 340.0747.

\section{0a (trans-3,5-diethoxy-2'-fluoro-4'-methoxy stilbenes)}

White solid, yield $28 \%$, m.p. $57.3^{\circ} \mathrm{C} \sim 58.2^{\circ} \mathrm{C}$. IR $v_{\max }\left(\mathrm{cm}^{-1}\right)$, 835, 962, 1106, 1170, 1290, 1444, 1506, 1532, 1619.2933, 2979. ${ }^{19} \mathrm{~F}$ NMR (376 MHz, $\left.\mathrm{CDCl}_{3}\right) \delta-115.55$ (dd, $\left.J=12.8,8.7 \mathrm{~Hz}\right) .{ }^{1} \mathrm{H}$ NMR $\left(400 \mathrm{MHz}, \mathrm{CDCl}_{3}\right) \delta$ 7.49 (t, $J=8.7 \mathrm{~Hz}, 1 \mathrm{H}), 7.15$ (d, $J=16.4 \mathrm{~Hz}, 1 \mathrm{H}), 6.95$ (d, $J=16.4 \mathrm{~Hz}, 1 \mathrm{H}), 6.70$ (dd, $J=8.7,2.4 \mathrm{~Hz}, 1 \mathrm{H}), 6.63$ (dd, $J=12.8,2.3 \mathrm{~Hz}, 3 \mathrm{H}), 6.37$ (t, $J=2.3 \mathrm{~Hz}, 1 \mathrm{H}), 4.05$ (q, $J=7.0 \mathrm{~Hz}, 4 \mathrm{H}), 3.81$ (s, 3H), 1.42 (t, $J=7.0 \mathrm{~Hz}$, 6H). ${ }^{13} \mathrm{C}$ NMR (101 MHz, $\left.\mathrm{CDCl}_{3}\right) \delta 162.30(\mathrm{~s}), 160.25$ (t, $\left.J=5.2 \mathrm{~Hz}\right), 159.82(\mathrm{~s}), 139.46$ (s), 128.63 (d, $J=4.7$ $\mathrm{Hz}$ ), 127.61 (d, $J=5.6 \mathrm{~Hz}$ ), 121.03 (d, $J=3.1 \mathrm{~Hz}$ ), 117.63 (d, $J=12.6 \mathrm{~Hz}$ ), 110.46 (d, $J=2.9 \mathrm{~Hz}$ ), 104.98 (s), 101.57 (d, $J=26.0 \mathrm{~Hz}$ ), 100.79 (s), 63.49 (s), 55.56 (s), 14.85 (s). HRMS(EI), Calcd. for $\mathrm{C}_{19} \mathrm{H}_{21} \mathrm{O}_{3} \mathrm{~F}, 316.1475$, Found, 316.1469 .

\section{$10 \mathrm{~b}$ (trans-3,5-n-dipropoxy-2'-fluoro-4'-methoxy stilbenes)}

White solid, yield $21 \%$, m.p. $58.3^{\circ} \mathrm{C} \sim 60.2^{\circ} \mathrm{C}$. IR $v_{\max }\left(\mathrm{cm}^{-1}\right)$, 831, 962, 1066, 1197, 1290, 1445, 1506, 1596, 1619, 2876, 2964. ${ }^{19} \mathrm{~F}$ NMR (376 MHz, $\left.\mathrm{CDCl}_{3}\right) \delta-115.55$ (dd, $\left.J=12.4,8.7 \mathrm{~Hz}\right) .{ }^{1} \mathrm{H}$ NMR $\left(400 \mathrm{MHz}, \mathrm{CDCl}_{3}\right) \delta$ 7.49 (t, $J=8.7 \mathrm{~Hz}, 1 \mathrm{H}), 7.16$ (d, $J=16.5 \mathrm{~Hz}, 1 \mathrm{H}), 6.96$ (d, $J=16.5 \mathrm{~Hz}, 1 \mathrm{H}), 6.70$ (dd, $J=8.7,2.4 \mathrm{~Hz}, 1 \mathrm{H}), 6.63$ (dd, $J=12.4,2.2 \mathrm{~Hz}, 3 \mathrm{H}), 6.38$ (t, $J=2.2 \mathrm{~Hz}, 1 \mathrm{H}), 3.94(\mathrm{t}, J=6.6 \mathrm{~Hz}, 4 \mathrm{H}), 3.81$ (s, 3H), $1.88-1.75$ (m, 4H), 1.04 (t, $J=7.4 \mathrm{~Hz}, 6 \mathrm{H}) .{ }^{13} \mathrm{C}$ NMR (101 MHz, $\left.\mathrm{CDCl}_{3}\right) \delta 162.30$ (s), 160.45 (s), 160.23 (d, $\left.J=11.2 \mathrm{~Hz}\right), 159.82$ 
(s), 139.42 (s), 128.68 (d, $J=4.7 \mathrm{~Hz}), 127.61$ (d, $J=5.6 \mathrm{~Hz}), 121.00$ (d, $J=3.1 \mathrm{~Hz}), 117.66$ (d, $J=12.6 \mathrm{~Hz})$, 110.46 (d, $J=2.9 \mathrm{~Hz}$ ), 104.97 (s), 101.57 (d, $J=26.0 \mathrm{~Hz}$ ), 100.82 (s), 69.57 (s), 55.57 (s), 22.62 (s), 10.56 (s). HRMS(EI), Calcd. for, $\mathrm{C}_{21} \mathrm{H}_{25} \mathrm{O}_{3} \mathrm{~F}$, 344.1788, Found, 344.1782.

10c (trans-4-ethoxy-3'-fluoro-4'-methoxy stilbenes)

White solid, yield $27 \%$, m.p. $160.0^{\circ} \mathrm{C} \sim 160.6^{\circ} \mathrm{C}$. IR $v_{\max }\left(\mathrm{cm}^{-1}\right), 526,649,734,909,1025,1161,1285,1442$, 1517, 1604, 2842, 2983. ${ }^{19} \mathrm{~F}$ NMR (376 MHz, $\left.\mathrm{CDCl}_{3}\right) \delta-135.43$ (dd, $J=12.6,8.8 \mathrm{~Hz}$ ). ${ }^{1} \mathrm{H}$ NMR (400 MHz, $\left.\mathrm{CDCl}_{3}\right) \delta 7.41(\mathrm{~d}, J=8.8 \mathrm{~Hz}, 1 \mathrm{H}), 7.24(\mathrm{~d}, J=1.9 \mathrm{~Hz}, 1 \mathrm{H}), 7.15(\mathrm{~d}, J=8.4 \mathrm{~Hz}, 1 \mathrm{H}), 6.94(\mathrm{~d}, J=8.4 \mathrm{~Hz}, 1 \mathrm{H})$, $6.90(\mathrm{~d}, J=2.0 \mathrm{~Hz}, 1 \mathrm{H}), 6.87(\mathrm{~d}, J=2.0 \mathrm{~Hz}, 1 \mathrm{H}), 4.06$ (q, $J=7.0 \mathrm{~Hz}, 2 \mathrm{H}), 3.91$ (s, 3H), 1.43 (t, $J=7.0 \mathrm{~Hz}$, 3H).13C NMR (101 MHz, cdcl3) $\delta 158.63$ (s), 153.78 (s), 151.34 (s), 146.82 (d, $J=11.1 \mathrm{~Hz}), 131.34$ (d, $J=6.6$ Hz), 129.77 (s), 127.55 (d, $J=2.4 \mathrm{~Hz}$ ), 125.00 (d, $J=2.4 \mathrm{~Hz}$ ), 122.53 (d, $J=3.3 \mathrm{~Hz}$ ), 114.67 (s), 113.35 (d, $J=$ $2.3 \mathrm{~Hz}$ ), 113.08 (d, $J=18.7 \mathrm{~Hz}$ ), 63.47 (s), 56.29 (s), 14.81 (s). HRMS(EI), Calcd. for $\mathrm{C}_{17} \mathrm{H}_{17} \mathrm{O}_{2} \mathrm{~F}, 272.1213$, Found, 272.1215.

\subsection{Anti-Cancer Assays}

The in vitro anti-cancer activities of flourine-substituted resveratrol derivatives were studied on human cells breast carcinoma (MDA-MB-231) and gastric carcinoma cell lines (SGC-7901) by applying the MTT assay as described by Mosmann [20]. Briefly, cells were seeded at a density of $10^{4}$ cells/well in 96-well microtiter plates and incubated in $5 \% \mathrm{CO}_{2}$ at $37^{\circ} \mathrm{C}$ for $24 \mathrm{~h}$. The tested compounds at indicated concentrations were added to culture medium, and the cell cultures were continued for another $48 \mathrm{~h}$. After $48 \mathrm{~h}$, cell survival was determined by the addition of an MTT solution (5 mg/mL MTT in PBS). We calculated optical density (OD) at $570 \mathrm{~nm}$ with EX-800 Eliasa $\mathrm{IC}_{50}$, calculated by OD, was used to evaluate the effect on the cell proliferation. All of the compounds were tested three times in each of the cell lines.

\subsection{Anticancer Activity}

MTT assay is dependent on $\mathrm{NAD}(\mathrm{P}) \mathrm{H}$-dependent oxidoreductase enzymes largely in the cytosolic compartment of the cell [21]. Therefore, reduction of MTT depends on the cellular metabolic activity due to NAD(P)H flux. In vitro MTT assays were done through the same method as previously work described [22]. The synthesized compounds 5a 5c and 10a 10c were evaluated by MTT-based assay using breast cancer cell lines (MDAMB-231) and gastric carcinoma cell lines (SGC-7901) with 5-FU and resveratrol as the positive control. The $\mathrm{IC}_{50}$ represents the concentration of a drug that can induce the death of $50 \%$ cancer cells in vitro. The given values are mean values of three experiments (Table 1 ).

As summarized in Table 1, these synthetic resveratrol derivatives exhibited a remarkable different inhibitory activity against two cancer cell lines. Compound 5c shows the strongest activity against breast carcinoma (MDA-MB-231) and gastric carcinoma cell lines (SGC-7901) with $\mathrm{IC}_{50}$ value of 50.19 $\pm 1.02 \mu \mathrm{M}, 122.68 \pm 2.04$

Table 1. The anticancer activities of resveratrol derivatives in vitro.

\begin{tabular}{cccc}
\hline Compound & Molecular weight & \multicolumn{2}{c}{ IC $_{50}(\boldsymbol{\mu M})$} \\
\cline { 3 - 3 } & & \multicolumn{1}{c}{ MDA-231 } & $184.54 \pm 1.24$ \\
$\mathbf{5 a}$ & 288.11 & $66.23 \pm 0.96$ & -- \\
$\mathbf{5 b}$ & 288.11 & $51.27 \pm 0.84$ & $122.68 \pm 2.04$ \\
$\mathbf{5 c}$ & 340.17 & $50.19 \pm 1.02$ & $284.5 \pm 2.36$ \\
$\mathbf{1 0 a}$ & 316.14 & $440.22 \pm 0.78$ & -- \\
$\mathbf{1 0 b}$ & 344.17 & $57.08 \pm 0.32$ & -- \\
$\mathbf{1 0 c}$ & 272.12 & $64.06 \pm 0.27$ & $156.74 \pm 2.64$ \\
$\mathbf{5 - F U}$ & 262.19 & $98.59 \pm 0.74$ & $184.3 \pm 1.38$ \\
\hline
\end{tabular}

$\mathrm{IC}_{50}=$ compound concentration required to inhibit tumor cell proliferation by $50 \%$. Data are expressed as the mean \pm SE from the dose-response curves of at least three independent experiments. ---, promotes the growth of cancer cells. 
$\mu \mathrm{M}$, compared to $\mathrm{IC}_{50}$ value of $5-\mathrm{FU}$ is $(98.59 \pm 3.61 \mu \mathrm{M}, 156.74 \pm 6.16 \mu \mathrm{M}$, respectively). Moreover, $5 \mathrm{c}$ is little better than resvertarol where the difference is about 3-fold. All the compounds except 10a have shown good anti-breast cancer activity than 5-FU and resveratrol which prove that these series of resveratrol derivatives possess visible anti-cancer activity.

\section{Conclusion}

In summary, $\mathbf{6}$ new resveratrol derivatives were successfully synthesized. Most of the synthetic compounds indicated higher activities than resveratrol and 5-FU. From the results, compound 5c was identified as the most effective candidate item against breast cancer and gastric carcinoma cells lines. It is expected that the pharmacological studies described in this article will promote the design of new therapeutic drugs for the clinical treatment of breast cancer and gastric carcinoma. It shows the potentiality as a therapeutic for humans.

\section{Acknowledgements}

This research was supported by the National Natural Science Foundation of China (No. 81273537), the key disciplines of Hunan Province, College Students’ Innovative Projects of Hunan Province, and the Zhengxiang Scholar Program of the University of South China.

\section{References}

[1] Chen, H., Tan, C. and Wu, T. (2011) Ensemble Modeling Coupled with Six Element Concentrations in Human Blood for Cancer Diagnosis. Biological Trace Element Research, 143, 143-152. http://dx.doi.org/10.1007/s12011-010-8864-1

[2] Siegel, R., Naishadham, D. and Jemal, A. (2013) Cancer Statistics. CA: A Cancer Journal for Clinicians, 63, 11-30. http://dx.doi.org/10.3322/caac.21166

[3] Cancer Facts and Figures 2014. American Cancer Society, Atlanta, GA.

[4] Jemal, A., Bray, F., Center, M.M., Ferlay, J., Ward, E. and Forman, D. (2011) Global Cancer Statistics. CA: A Cancer Journal for Clinicians, 61, 69-90. http://dx.doi.org/10.3322/caac.20107

[5] Nobili, S., Bruno, L., Landini, I., Napoli, C., Bechi, P., Tonelli, F., Rubio, C.A., Mini, E. and Nesi, G. (2011) Genomic and Genetic Alterations Influence the Progression of Gastric Cancer. World Journal of Gastroenterology, 17, $290-299$. http://dx.doi.org/10.3748/wjg.v17.i3.290

[6] de Souza, C.R., de Oliveira, K.S., Ferraz, J.J., Leal, M.F., Calcagno, D.Q., Seabra, A.D., Khayat, A.S., Montenegro, R.C., Alves, A.P., Assumpção, P.P., Smith, M. and Burbano, R.R. (2014) Occurrence of Helicobacter pylori and Epstein-Barr Virus Infection in Endoscopic and Gastric Cancer Patients from Northern Brazil. BMC Gastroenterology, 14, 179. http://dx.doi.org/10.1186/1471-230X-14-179

[7] Takaoka, M.J. (1940) Of the Phenolic Substances of White Hellebore (Veratrum grandiflorum Loes. fil.). Journal of the Faculty of Science, Hokkaido Imperial University, 3, 1-16.

[8] Wu, C.F., Yang, J.Y., Wang, F. and Wang, X.X. (2013) Resveratrol, Botanical Origin, Pharmacological Activity and Applications. Chinese Journal of Natural Medicines, 11, 1-15. http://dx.doi.org/10.3724/SP.J.1009.2013.00001

[9] Liu, W.B., Hu, L., Hu, Q., Chen, N.N., Yang, Q.S. and Wang, F.F. (2013) New Resveratrol Oligomer Derivatives from the Roots of Rheum lhasaense. Molecules, 18, 7093-7102. http://dx.doi.org/10.3390/molecules18067093

[10] Yang, X., Li, X. and Ren, J. (2014) From French Paradox to Cancer Treatment, Anti-Cancer Activities and Mechanisms of Resveratrol. Anti-Cancer Agents in Medicinal Chemistry, 14, 806-825. http://dx.doi.org/10.2174/1871520614666140521121722

[11] Sun, D., Hurdle, J.G., Lee, R., Cushman, M. and Pezzuto, J.M. (2012) Evaluation of Flavonoid and Resveratrol Chemical Libraries Reveals Ayssionon II as a Promising Antibacterial Lead. ChemMedChem, 7, 1541-1545. http://dx.doi.org/10.1002/cmdc.201200253

[12] Tresguerres, I.F., Tamimi, F., Eimar, H., Barralet, J., Torres, J., Blanco, L. and Tresguerres, J.A. (2014) Resveratrol as Anti-Aging Therapy for Age-Related Bone Loss. Rejuvenation Research, 17, 439-445. http://dx.doi.org/10.1089/rej.2014.1551

[13] Chen, X., Lu, J., An, M., Ma, Z., Zong, H. and Yang, J. (2014) Anti-Inflammatory Effect of Resveratrol on Adjuvant Arthritis Rats with Abnormal Immunological Function via the Reduction of Cyclooxygenase-2 and Prostaglandin E2. Molecular Medicine Reports, 9, 2592-2598. http://dx.doi.org/10.3892/mmr.2014.2070

[14] Yao, J., Wang, J.Y., Liu, L., Li, Y.X., Xun, A.Y., Zeng, W.S., Jia, C.H., Wei, X.X., Feng, J.L., Zhao, L. and Wang, L.S. (2010) Anti-Oxidant Effects of Resveratrol on Mice with DSS-Induced Ulcerative Colitis. Archives of Medical Re- 
search, 41, 288-294. http://dx.doi.org/10.1016/j.arcmed.2010.05.002

[15] Bhat, K.P.L., Kosmeder, J.W. and Pezzuto, J.M. (2001) Biological Effects of Resveratrol. Antioxidants \& Redox Signaling, 3, 1041-1064. http://dx.doi.org/10.1089/152308601317203567

[16] Kopp, P. (1998) Resveratrol a Phytoestrogen Found in Red Wine. A Possible Explanation for the Conundrum of the French Paradox? European Journal of Endocrinology, 138, 619-620. http://dx.doi.org/10.1530/eje.0.1380619

[17] Das, S. and Ng, K.Y. (2011) Quantification of Trans-Resveratrol in Rat Plasma by a Simple and Sensitive High Performance Liquid Chromatography Method and Its Application in Pre-Clinical Study. Journal Of Liquid Chromatography \& Related Technologies, 34, 1399-1414. http://dx.doi.org/10.1080/10826076.2011.572215

[18] Marier, J.F., Vachon, P., Gritsas, A., Zhang, J., Moreau, J.P. and Ducharme, M.P. (2002) Metabolism and Disposition of Resveratrol in Rats, Extent of Absorption, Glucuronidation, and Enterohepatic Recirculation Evidenced by a Linked-Rat Model. Journal of Pharmacology and Experimental Therapeutics, 302, 369-373. http://dx.doi.org/10.1124/jpet.102.033340

[19] Sun, B., Hoshino, J., Jermihov, K., Marler, L., Pezzuto, J.M., Mesecar, A.D. and Cushman, M. (2010) Design, Synthesis, and Biological Evaluation of Resveratrol Analogues as Aromatase and Quinone Reductase 2 Inhibitors for Chemoprevention of Cancer. Bioorganic \& Medicinal Chemistry, 18, 5352-5366. http://dx.doi.org/10.1016/j.bmc.2010.05.042

[20] Mosman, T. (1983) Rapid Colorimetric Assay for Cellular Growth and Survival, Application to Proliferation and Cytotoxicity Assays. Journal of Immunological Methods, 65, 55-63. http://dx.doi.org/10.1016/0022-1759(83)90303-4

[21] Berridge, M.V., Herst, P.M. and Tan, A.S. (2005) Tetrazolium Dyes as Tools in Cell Biology, New Insights into Their Cellular Reduction. Biotechnology Annual Review, 11, 127-152. http://dx.doi.org/10.1016/S1387-2656(05)11004-7

[22] Zheng, X., Yu, L., Yang, J., Yao, X., Yan, W., Bo, S., Liu, Y., Wei, Y., Wu, Z. and Wang, G. (2014) Synthesis and Anti-Cancer Activities of Apigenin Derivatives. Medicinal Chemistry, 10, 747-752. http://dx.doi.org/10.2174/1573406410666140307152557

\section{Submit or recommend next manuscript to SCIRP and we will provide best service for you:}

Accepting pre-submission inquiries through Email, Facebook, LinkedIn, Twitter, etc. A wide selection of journals (inclusive of 9 subjects, more than 200 journals)

Providing 24-hour high-quality service

User-friendly online submission system

Fair and swift peer-review system

Efficient typesetting and proofreading procedure

Display of the result of downloads and visits, as well as the number of cited articles

Maximum dissemination of your research work

Submit your manuscript at: http://papersubmission.scirp.org/

Or contact ojmc@scirp.org 